\title{
Strom à la carte - Das Menü eines innovativen Energieversorgers
}

\section{Digitalisierung - Irritation - Innovation}

\author{
L. Schitter
}

Online publiziert am 28. November 2017

(C) Springer-Verlag GmbH Austria, ein Teil von Springer Nature 2017

Die Energiewirtschaft befindet sich in einem enormen Umbruch. Dem vorweg steht der Klimawandel.

Der Klimawandel ist für die Wirtschaft sowohl Irritation als auch Motor. Wir sind in jedem Fall gezwungen, rasch zu handeln. Neueste Studien amerikanischer Forscher zeigen, dass - wenn wir nichts unternehmen - die Temperaturen mit hoher Wahrscheinlichkeit noch in diesem Jahrhundert nicht wie geplant um max. $2{ }^{\circ} \mathrm{C}$, sondern um $3,2^{\circ} \mathrm{C}$ steigen werden. Es gibt Handlungsbedarf, denn der Klimawandel ist keine Glaubensfrage - konkrete Maßnahmen sind gefragt. Nur wer sich mit Megatrends wie Dezentralisierung, Dekarbonisierung und - derzeit vor allem - Digitalisierung beschäftigt, wird den Klimawandel meistern können.

Um die - bewusst gewählt - „Umwälzung" der Energiewirtschaft anzugehen, braucht es neue Lösungswege. Die Digitalisierung als Innovationsmotor ist nicht mehr wegzudenken, sie ermöglicht uns, die volatilen neuen Energien in das Stromsystem zu integrieren und schafft neue Angebote und Services: Drohnen werden für die Überprüfung von Stromleitungen eingesetzt, digitale Medien bieten neue Möglichkeiten für den Kundenkontakt, Smart Meter und Smart Home-Produkte unterstützen unsere Kunden dabei, Energie effizienter einzusetzen. Alles, was digitalisiert werden kann, wird digitalisiert werden. Und alles, was digitalisiert wird, braucht Strom. Die E-Wirtschaft betrachtet Investitionssicherheit als unverzichtbare Grundlage des anstehenden Umbaus des Stromsystems. Digitalisierung ist keine Investition in ein Kraftwerk im herkömmlichen Sinn.

Für innovative Energieversorger bestimmt der Klimawandel die Strategie. In der E-Wirtschaft haben wir nicht nur betriebswirtschaftliche Aspekte zu beachten, sondern Daseinsvorsorge für heute und auch für die Zukunft Österreichs zu leisten.

Für uns ist klar: Erneuerbare Energien schonen die Ressourcen am meisten. Dass Investition in erneuerbare Energie zulasten der Profitabilität geht, muss dabei in Kauf genommen werden

Österreich hat - auch aufgrund seiner Topologie und der Leistungen unserer Vorgänger beim Ausbau der Wasserkraft - eine hervorragende Ausgangssituation. Damit diese gehalten werden kann, braucht es aber mehr Tempo im Ausbau der Stromerzeugung aus erneuerbaren Energien.

Seriös zu informieren, aufzuklären und das Vertrauen der Anrainer bei Kraftwerksprojekten zu gewinnen, ist hier auch eine wesentliche Aufgabe der Energiewirtschaft.

Als Energieversorger haben wir natürlich auch den unternehmerischen Auftrag, eine Versorgungssicherheitsoffensive zu starten und damit weiterhin 100\% Versorgungssicherheit zu garantieren. Daher müssen wir unsere Netze massiv ausbauen, und zwar Übertragungs- wie Verteilernetze. Das soll allen Kunden und so auch den produzierenden Konsumenten zugutekommen. Eine Verankerung der Netzbetreiber als zentrale Datendrehscheibe für das zukünftige Informations- und Datenmanagement ist für uns ebenso eine Selbstverständlichkeit wie flexible, systemrelevante Kraftwerke, die im Falle schwankender Erzeugungsleistung die Systemsicherheit garantieren.

\section{Investition in Infrastruktur schafft Wertschöpfung!}

Ein Gelingen des Paradigmenwechsels beim Ausbau erneuerbarer Energien bedeutet auch einen immensen Impuls für die heimische Wirtschaft. Bis 2030 sind bis zu 50 Milliarden Euro an Investitionen in ein modernes Energiesystem in Österreich möglich. Dieses enorme Infrastrukturpaket bringt Wertschöpfung im ganzen Land, denn rund 30.000 direkte und indirekte Arbeitsplätze werden damit geschaffen bzw. erhalten. Davon profitiert ganz Österreich.

Fazit: Unsere Branche trifft der Klimawandel so stark wie kaum eine andere. Klima und Klimawandel sind Themen, die für uns von großer Bedeutung sind, denn wir leben davon und sind davon abhängig. Energiewende braucht Strom, Innovation und Investition. Schließlich muss es unsere klare strategische Ausrichtung sein, unseren Kunden Strom aus erneuerbaren Energiequellen zu liefern. Hier gilt es, Modelle zu entwickeln, um die nötigen Investitionen tätigen zu können. Die E-Wirtschaft wird ihren Beitrag zum Gelingen der Energiewende verantwortungsvoll und aktiv leisten. Nur gemeinsam können wir es schaffen!
Kurzfassung eines Vortrags bei der 55. Fachtagung der Österreichischen Gesellschaft für Energietechnik (OGE) im OVE, die am 12. und 13. Oktober 2017 in Salzburg stattfand.

Schitter, Leonhard, Salzburg AG für Energie, Verkehr und Telekommunikation, Bayerhamerstraße 16, 5020 Salzburg, Österreich

(E-Mail: leonhard.schitter@salzburg-ag.at) 